\title{
Mapping interactome networks of DNAJC11, a novel mitochondrial protein causing neuromuscular pathology in mice
}

Foteini Violitzi ${ }^{1,2}$, Vasiliki-Iris Perivolidi ${ }^{1,2}$, Trias Thireou $^{1}$, Ioannis Grivas $^{3}$, Sylva Haralambous $^{3}$, Martina Samiotaki $^{2}$, George Panayotou$^{2}$, Eleni Douni ${ }^{1,2, *}$

${ }^{1}$ Laboratory of Genetics, Department of Biotechnology, Agricultural University of Athens, Iera Odos 75, 11855, Athens, Greece

${ }^{2}$ Institute for Bioinnovation, Biomedical Sciences Research Center "Alexander Fleming", Fleming 34, 16672, Vari, Greece

${ }^{3}$ Transgenic Technology Lab and Inflammation Research Group, Hellenic Pasteur Institute, Vas.Sofias 127, 11521, Athens, Greece

${ }^{*}$ Corresponding author: Eleni Douni, Laboratory of Genetics, Department of Biotechnology, Agricultural University of Athens, Iera Odos 75, 11855 Athens, Greece; douni@aua.gr

\section{SUPPORTING INFORMATION}




\section{$\underline{\text { Table of contents }}$}

-Suppl. Figure S1: Functional clustering of DNAJC11 protein interactors in HEK293FT cells.

-Suppl. Figure S2: DNAJC11 co-immunoprecipites with HSPA1A-MYC in HEK293FT cells.

-Suppl. Figure S3: Characterization of Tg704DNAJC11-FLAG transgenic mice.

-Suppl. Figure S4: Functional clustering of DNAJC11 protein interactors identified in the mouse cerebrum.

-Suppl. Table S1: LFQ values for proteins that co-immunoprecipitated with DNAJC11 ${ }^{\text {WT }}$ FLAG in HEK293 cells identified by LC-MS/MS analysis after quantification with MaxQuant program in gene name alphabetical order.

-Suppl. Table S2: The p-value and $\log 2$ difference of proteins immunoprecipitating with DNAJC $11^{\text {WT }}$-FLAG in HEK293 cells after statistical analysis with Perseus software in difference descending order for each cluster.

-Suppl. Table S3: Cellular component content and biological process content of putative DNAJC11 interactors in HEK293 cells grouped in Enrichment analysis of Cytoscape using the ClueGO/CluePedia plugins, presented in Figure 1D-E.

-Suppl. Table S4: MiST scoring of proteins immunoprecipitating with DNAJC1 ${ }^{\text {WT }}$-FLAG in HEK293 cells is given in score descending order.

-Suppl. Table S5: LFQ values for proteins that co-immunoprecipitated with DNAJC11 ${ }^{\triangle \mathrm{DUF}}$ FLAG in HEK293 cells identified by LC-MS/MS analysis after quantification with MaxQuant program in gene name alphabetical order.

-Suppl. Table S6: The p-value and $\log 2$ difference of proteins immunoprecipitating with DNAJC11 ${ }^{\triangle \mathrm{DUF}}$-FLAG in HEK293 cells after statistical analysis with Perseus software in difference descending order for each cluster.

-Suppl. Table S7: MiST scoring of proteins immunoprecipitating with DNAJC11 ${ }^{\triangle \mathrm{DUF}}{ }_{\text {-FLAG in }}$ HEK293 cells is given in score descending order.

-Suppl. Table S8: LFQ values for proteins that co-immunoprecipitated with DNAJC11-FLAG in cerebrum of $\operatorname{Tg} 701$ mice identified by LC-MS/MS analysis after quantification with MaxQuant program in gene name alphabetical order.

-Suppl. Table S9: The p-value and $\log 2$ difference of proteins immunoprecipitating with DNAJC11-FLAG in cerebrum of Tg701 mice after statistical analysis with Perseus software in difference descending order for each cluster.

-Suppl. Table S10: Cellular component content and biological process content of putative DNAJC11 interactors in the cerebrum of $\mathrm{Tg} 701$ mice grouped in Enrichment analysis of Cytoscape using the ClueGO/CluePedia plugins, presented in Figure 5C-D.

-Suppl. Table S11: MiST scoring of proteins immunoprecipitating with DNAJC11-FLAG in cerebrum of $\mathrm{Tg} 701$ mice is given in score descending order. 


\section{Supplementary Figure S1}

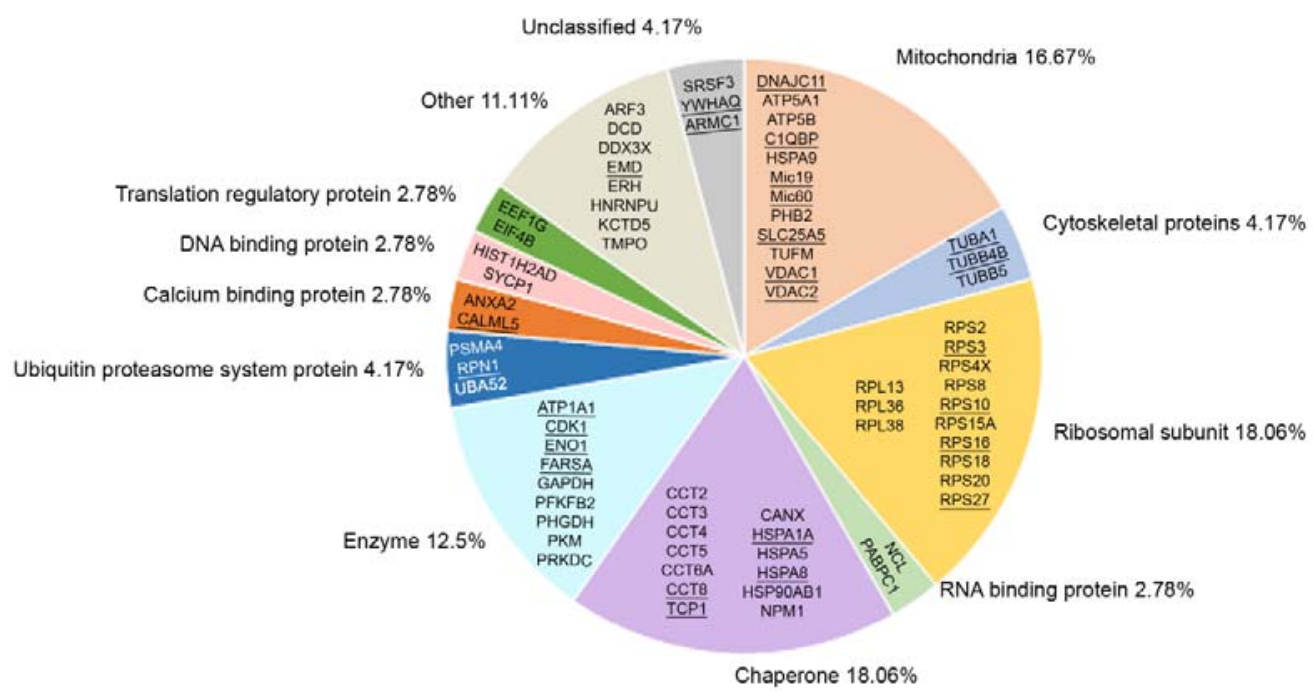

Supplementary Figure S1: Functional clustering of DNAJC11 protein interactors in

HEK293FT cells. Proteins co-immunoprecipitated in HEK293FT cells expressing DNAJC11FLAG protein were classified based on the molecular class according to the Human Protein Reference Database. Top DNAJC11 interactors confirmed by MiST scoring are underlined. 


\section{Supplementary Figure S2}

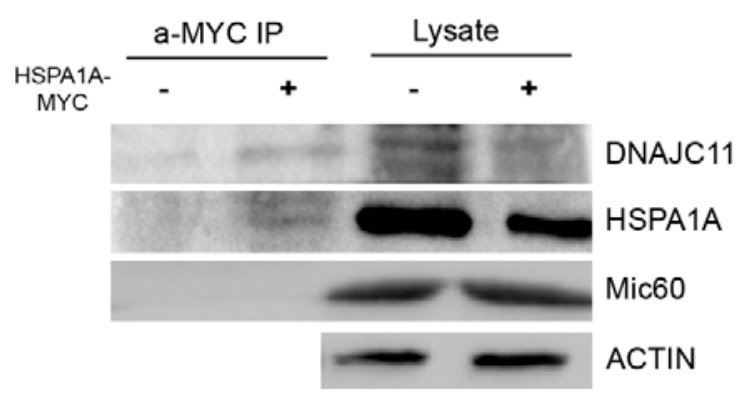

Supplementary Figure S2: DNAJC11 co-immunoprecipites with HSPA1A-MYC in HEK293FT cells. Representative western blot analysis on HEK293FT cell extracts expressing HSPA1A-MYC or not upon lysis and immunoprecipitation with beads coated with anti-MYC antibody (IP: a-MYC). Actin was used as loading control. Three independent experimental repeats were used in Western blot. 


\section{Supplementary Figure S3}

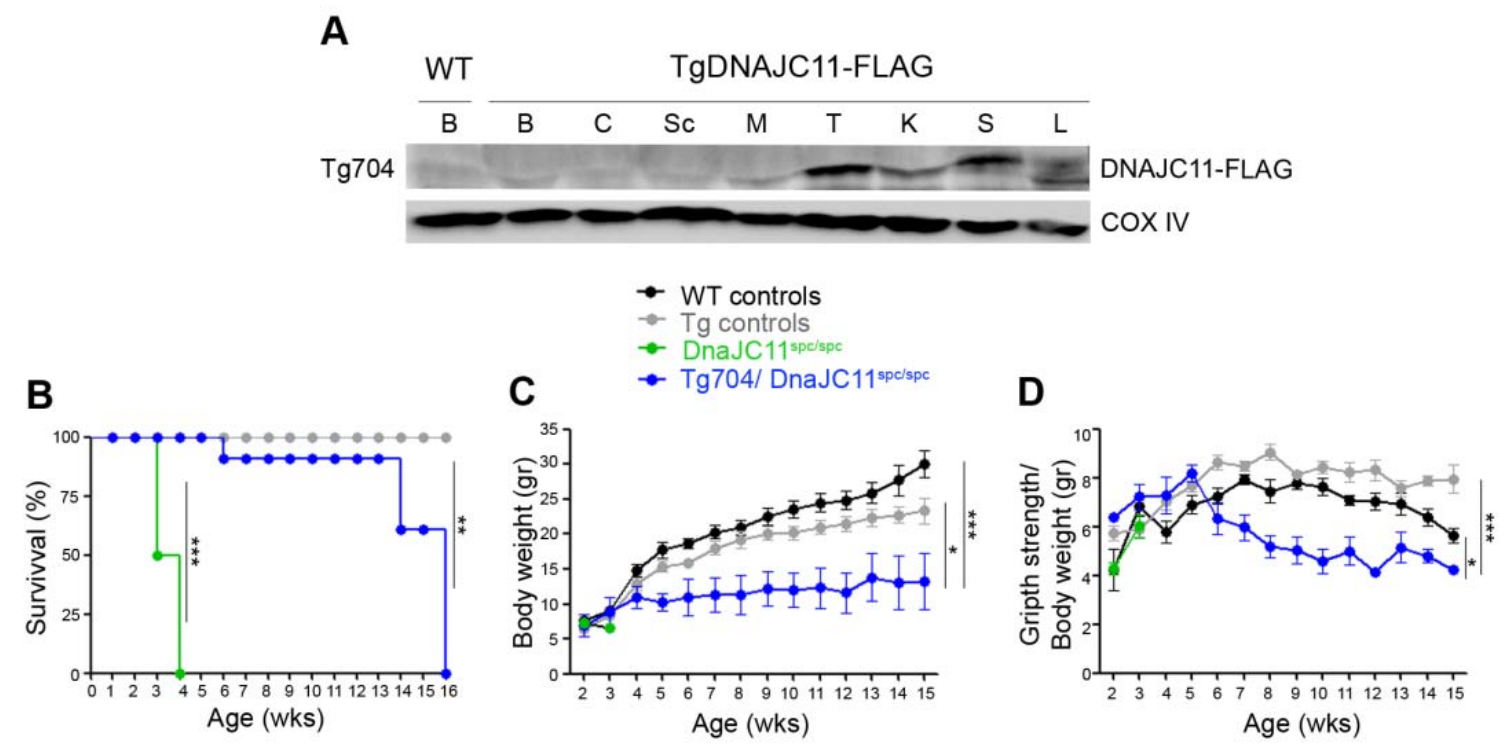

Supplementary Figure S3: Characterization of Tg704 DNAJC11-FLAG transgenic mice.

A) Expression pattern of the DnaJC11-FLAG transgene in mitochondrial protein extracts from cerebrum (B), cerebellum (C), spinal cord (Sc), muscle (M), thymus $(T)$, kidney $(K)$, spleen $(\mathrm{S})$ and liver (L) of $\operatorname{Tg} 704$ mice through western blot with an antibody against the FLAG peptide (DNAJC11-FLAG). Detection of COXIV was used as a loading control. WT cerebrum served as a negative control for FLAG detection. Rescue of B) premature lethality, C) body weight gain and D) muscle weakness in DnaJC1 $11^{\mathrm{spc} / \mathrm{spc}}$ mice expressing DNAJC11-FLAG. Incomplete rescue of the neuromuscular symptoms in Tg704/DnaJC1 $1^{\mathrm{spc} / \mathrm{spc}}$ mice (blue), compared to WT (black), Tg controls (Tg701 and Tg704, grey) and DnaJC11 ${ }^{\mathrm{spc} / \mathrm{spc}}$ mice (green) ( $\mathrm{n}=10$ per genotype). The log-rank test was used for survival curve comparison, where DnaJC11 ${ }^{\mathrm{spc} / \mathrm{spc}}$ mice showed a statistical difference compared to all other genotypes $(* * * \mathrm{p}<0.001)$, while Tg704/DnaJC11 $1^{\mathrm{spc} / \mathrm{spc}}$ mice also differed statistically from the WT and Tg701/704 mice $(* * p<0.01)$. One-Way ANOVA and Tukey post-hoc test was performed for statistical analysis in body weight curve and 
grip strength at the 15 th week of age. Data represent mean values \pm SEM. Asterisks mark statistically significant difference $(* \mathrm{p}<0.05, * * \mathrm{p}<0.01, * * * \mathrm{p}<0.001)$. 


\section{Supplementary Figure S4}

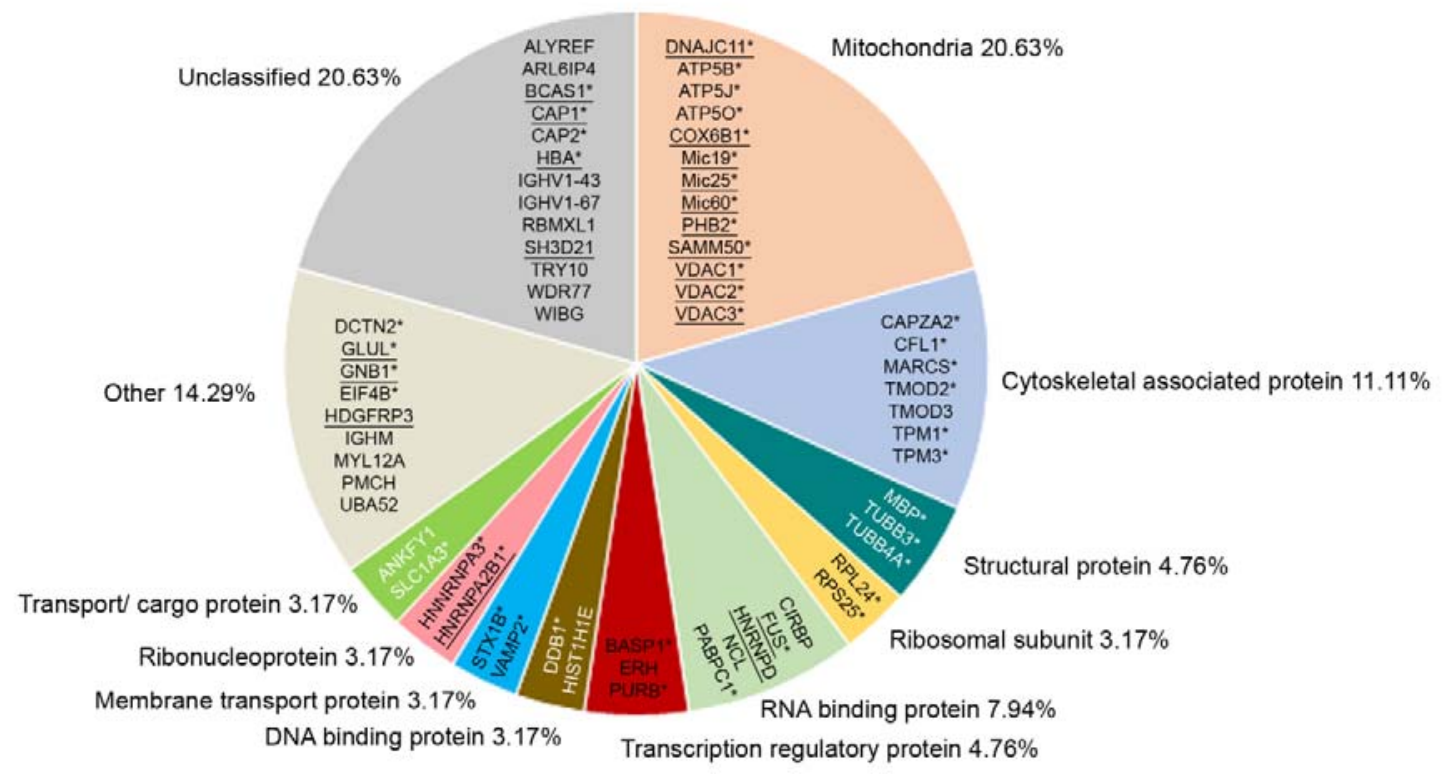

Supplementary Figure S4: Functional clustering of DNAJC11 protein interactors identified in the mouse cerebrum. Proteins co-immunoprecipitated in TgDNAJC11-FLAG cerebrum were classified based on the molecular class according to the Human Protein Reference Database. Top DNAJC11 interactors confirmed by MiST scoring are underlined. Asterisk denotes proteins identified as constituents of mouse brain cortical synapses ${ }^{48}$. 